\title{
Erratum: Effect of critical fluctuations on the spin transport in liquid ${ }^{3} \mathrm{He}$ [Phys. Rev. B 100, 094533 (2019)]
}

\author{
V. P. Mineev
}

(Received 17 December 2019; published 31 December 2019)

DOI: $10.1103 /$ PhysRevB.100.249902

The integration in Eq. (9) must be not over $d^{3} k$ but over $d^{3} p$,

$$
\Lambda_{\mathbf{p}, \mathbf{q}, \varepsilon_{n}, \Omega_{k}-\varepsilon_{n}}^{l}=\hat{p}_{l}+\frac{1}{2 \pi N_{o} \tau} \int \frac{d^{3} p}{(2 \pi)^{3}} G_{\mathbf{p}, \varepsilon_{n}} G_{-\mathbf{p}+\mathbf{q}, \Omega_{k}-\varepsilon_{n}} \Lambda_{\mathbf{p}, \mathbf{q}, \varepsilon_{n}, \Omega_{k}-\varepsilon_{n}}^{l} .
$$

This is a purely typographical error.

Equation (11) contains factor $i$ in the first term in the bracket missed in the original paper. The correct equation should be as follows:

$$
N_{0} \int d \xi G_{\mathbf{p}, \varepsilon_{n}} G_{\mathbf{p}, \varepsilon_{n}} G_{\mathbf{q}-\mathbf{p},-\varepsilon_{n}}=-\frac{\pi N_{0}}{2}\left(i \frac{\operatorname{sgn} \tilde{\varepsilon}_{n}}{\tilde{\varepsilon}_{n}^{2}}+\frac{(\mathbf{q} \cdot \mathbf{v})}{\left|\tilde{\varepsilon}_{n}\right|^{3}}\right) .
$$

This is a purely typographical error and does not affect any other part of the paper or the analysis.

Equation (12) contains the additional term missed in the original paper. The correct equation should be as follows:

$$
\begin{aligned}
\mathbf{B}_{i, \alpha \beta}^{l r}= & \frac{1}{60} \frac{N_{0} v_{F}^{2}}{(2 \pi T)^{2}}\left(\delta_{i l} q_{r}+\delta_{i r} q_{l}+\delta_{l r} q_{i}\right) \boldsymbol{\sigma}_{\alpha \beta} \psi^{\prime \prime}\left(\frac{1}{2}+\frac{1}{4 \pi T \tau}\right) \\
& -\frac{\pi}{36} N_{0} v_{F}^{2}\left(\delta_{i l} q_{r}+\delta_{i r} q_{l}\right) \boldsymbol{\sigma}_{\alpha \beta} T \sum_{n \geqslant 0} \frac{1}{\tilde{\varepsilon}_{n}^{3} \varepsilon_{n} \tau} .
\end{aligned}
$$

Equation (14) must be

$$
\epsilon=\ln \frac{T}{T_{c 0}}+\psi\left(\frac{1}{2}+\frac{1}{4 \pi T \tau}\right)-\psi\left(\frac{1}{2}\right)
$$

Taking into account the second term in Eq. (12), Eq. (19) must be

$$
\mathbf{B}_{i, \alpha \beta}^{l r}=-\frac{2}{3} N_{0} \xi^{2}\left(\delta_{i l} q_{r}+\delta_{i r} q_{l}+\delta_{l r} q_{i}\right) \sigma_{\alpha \beta}-\frac{2}{3} N_{0} \xi_{1}^{2}\left(\delta_{i l} q_{r}+\delta_{i r} q_{l}\right) \sigma_{\alpha \beta}
$$

where

$$
\xi_{1}^{2}= \begin{cases}\frac{5 \zeta(4)}{2^{7}} \frac{v_{F}^{2}}{(2 \pi T)^{2} 4 \pi T \tau}, & 4 \pi T \tau \gg 1, \\ \frac{1}{6} v_{F}^{2} \tau^{2} \ln \frac{e}{10 \pi T \tau}, & 4 \pi T \tau \ll 1 .\end{cases}
$$

Thus, in low scattering limit $4 \pi T \tau \gg 1$, one can neglect term $\propto \xi_{1}^{2}$ and Eqs. (22), (25), (27), and (28) are correct. Whereas in the ultra-low-temperature region $4 \gamma \frac{T}{T_{c}}<4 \pi T \tau \ll 1$, we have $\xi_{1}^{2}=\frac{5}{3} \xi^{2} \ln \frac{e}{10 \pi T \tau}$. Hence, in this temperature region in the classic (static) $\left(T-T_{c}\right) \ll T$ limit, the fluctuation current given by Eqs. (22), (25), (27), and (28) acquires factor $\propto\left(\ln \frac{e}{10 \pi T \tau}\right)^{2}$. The same is true with respect to Eq. (23) in the quantum region $\epsilon \gg T \tau$.

The sluggish logarithmic correction develops itself only at very low temperatures, therefore, it presents just academic interest. 